\title{
Surface Adsorbed Antibody Characterization Using ToF-SIMS with Principal Component Analysis and Artificial Neural Networks
}

Nicholas G. Welch ${ }^{\dagger, \dagger}$, Robert M. T. Madiona ${ }^{\dagger, \dagger}$, Thomas B. Payten ${ }^{\dagger}$, Robert T. Jones ${ }^{\dagger}$, Narelle Brack ${ }^{\dagger}$, Benjamin W. Muir ${ }^{\dagger}$, Paul J. Pigram *,ं

${ }^{\dagger}$ Centre for Materials and Surface Science and Department of Chemistry and Physics, School of Molecular Sciences, La Trobe University, Melbourne, VIC, 3086, Australia

†CSIRO Manufacturing, Clayton, VIC, 3168, Australia

\section{Corresponding Author}

*Email: p.pigram@latrobe.edu.au 

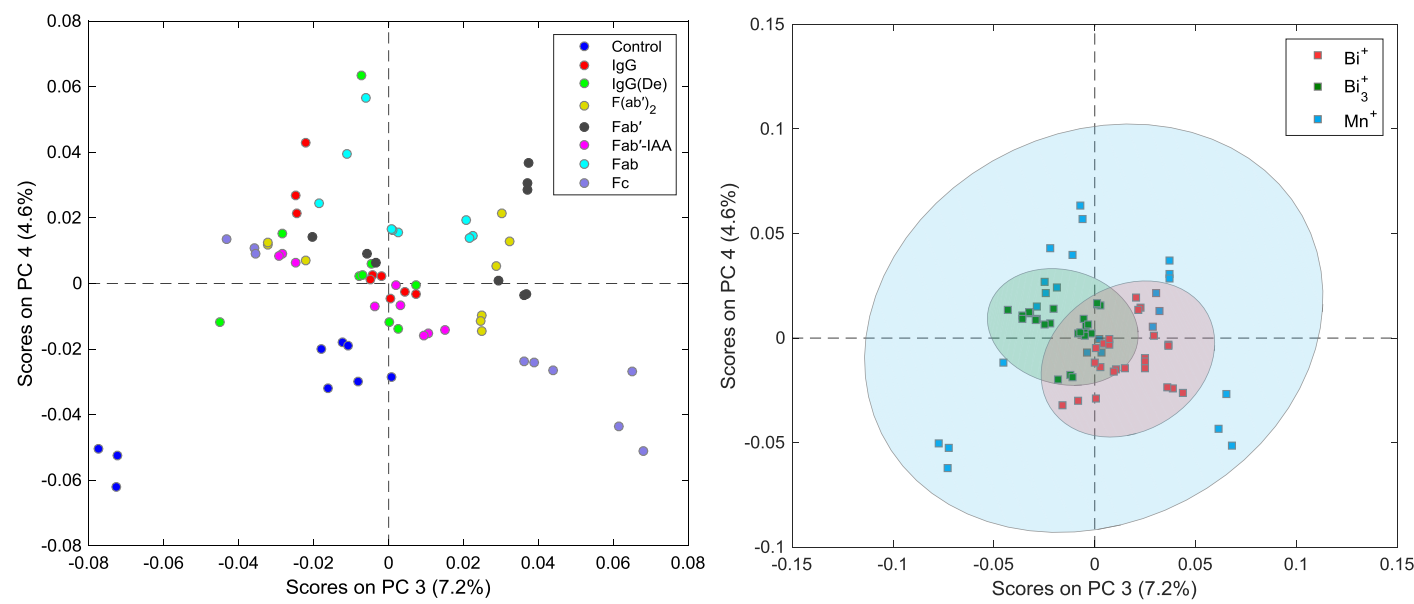

Figure S1. Principal component analysis of antibody fragments on silicon wafers PC 3 vs PC 4 as labeled by either protein fragment (left) or primary-ion source (right). ToF-SIMS peak list of 775 mass fragments was utilized. Ellipses show a $95 \%$ confidence level. 


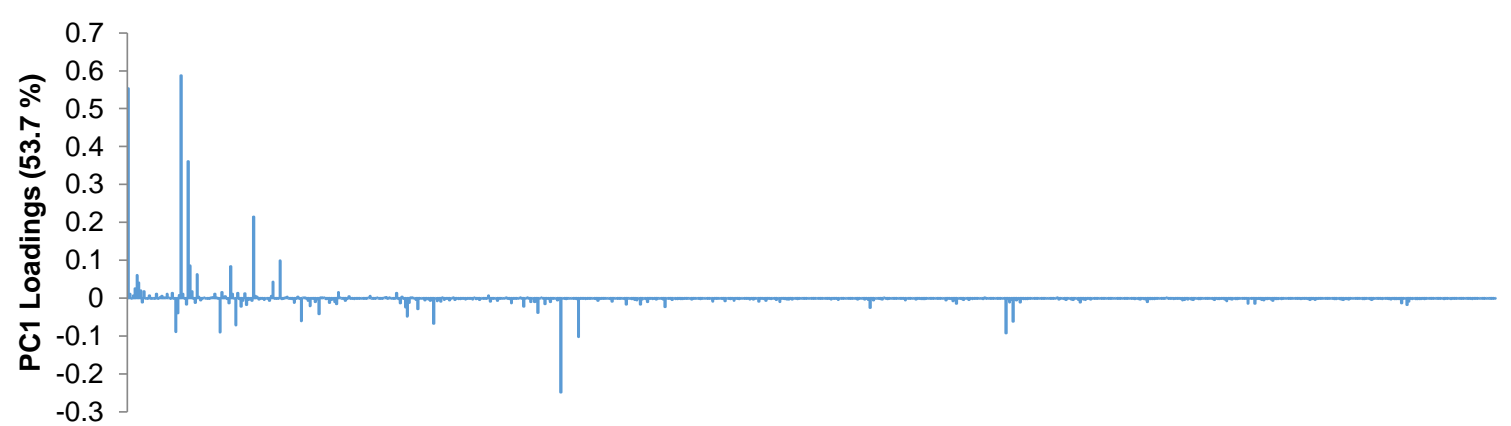

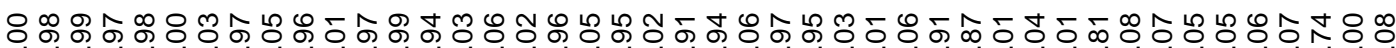

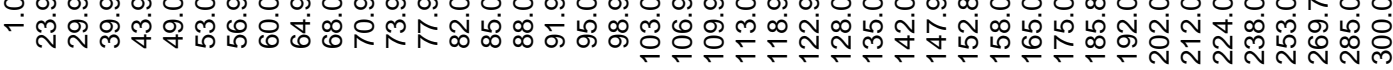

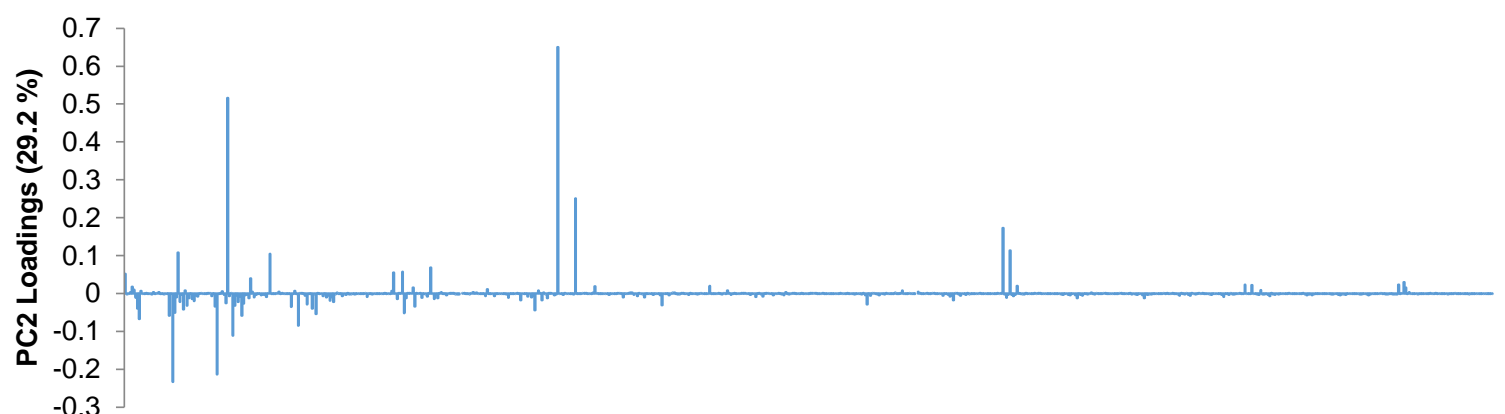

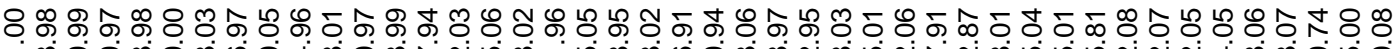

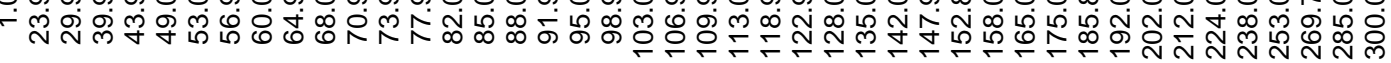
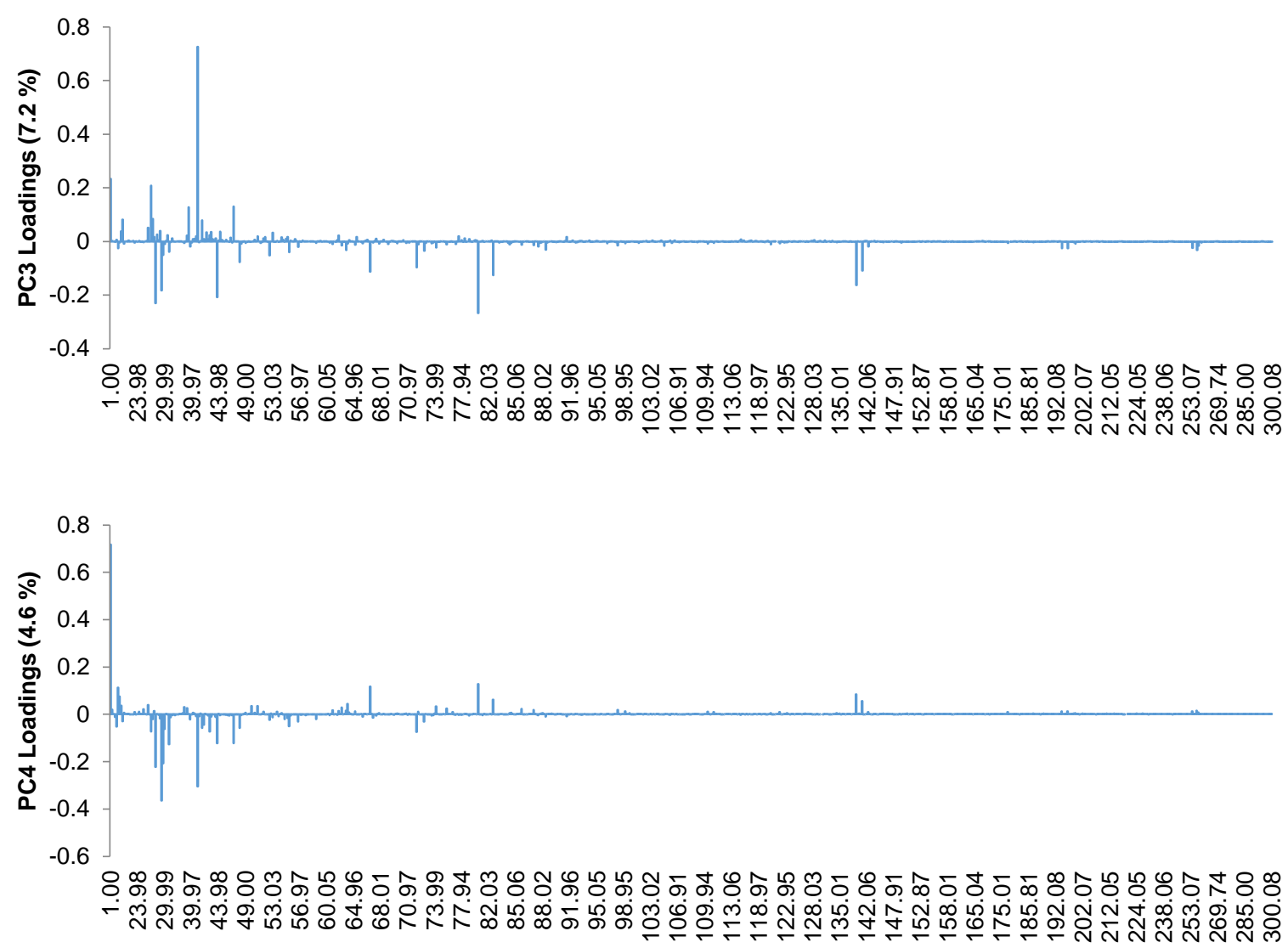

Fragment mass $(\mathrm{m} / \mathbf{z})$

Figure S2. Loadings plots for PCA of all samples utilizing 775 mass fragments. X-axis serves as a guide. 
Table S1. A compiled list of known ToF-SIMS mass fragments of amino acids.

\begin{tabular}{|c|c|c|c|}
\hline Number & $\mathrm{m} / \mathrm{z}$ & Fragment Label & Amino Acid \\
\hline 1 & 30.03 & $\mathrm{CH}_{4} \mathrm{~N}^{+}$ & glycine/lysine \\
\hline 2 & 43.03 & $\mathrm{CH}_{3} \mathrm{~N}_{2}^{+}$ & arginine \\
\hline 3 & 43.05 & $\mathrm{C}_{3} \mathrm{H}_{7}^{+}$ & isoleucine/leucine \\
\hline 4 & 44.01 & $\mathrm{CH}_{2} \mathrm{NO}^{+}$ & asparagine \\
\hline 5 & 44.05 & $\mathrm{C}_{2} \mathrm{H}_{6} \mathrm{~N}^{+}$ & alanine/lysine \\
\hline 6 & 56.05 & $\mathrm{C}_{3} \mathrm{H}_{6} \mathrm{~N}^{+}$ & lysine \\
\hline 7 & 60.05 & $\mathrm{C}_{2} \mathrm{H}_{6} \mathrm{NO}^{+}$ & serine \\
\hline 8 & 61.01 & $\mathrm{C}_{2} \mathrm{H}_{5} \mathrm{~S}^{+}$ & methionine \\
\hline 9 & 68.05 & $\mathrm{C}_{4} \mathrm{H}_{6} \mathrm{~N}^{+}$ & proline \\
\hline 10 & 69.03 & $\mathrm{C}_{4} \mathrm{H}_{5} \mathrm{O}^{+}$ & threonine \\
\hline 11 & 70.03 & $\mathrm{C}_{3} \mathrm{H}_{4} \mathrm{NO}^{+}$ & asparagine \\
\hline 12 & 70.07 & $\mathrm{C}_{4} \mathrm{H}_{8} \mathrm{~N}^{+}$ & arginine/proline/valine \\
\hline 13 & 71.01 & $\mathrm{C}_{3} \mathrm{H}_{3} \mathrm{O}_{2}^{+}$ & serine \\
\hline 14 & 72.04 & $\mathrm{C}_{3} \mathrm{H}_{6} \mathrm{NO}^{+}$ & alanine/threonine \\
\hline 15 & 72.08 & $\mathrm{C}_{4} \mathrm{H}_{10} \mathrm{~N}^{+}$ & valine \\
\hline 16 & 73.06 & $\mathrm{C}_{2} \mathrm{H}_{7} \mathrm{~N}_{3}{ }^{+}$ & arginine \\
\hline 17 & 74.06 & $\mathrm{C}_{3} \mathrm{H}_{8} \mathrm{NO}^{+}$ & threonine \\
\hline 18 & 80.05 & $\mathrm{C}_{5} \mathrm{H}_{6} \mathrm{~N}^{+}$ & proline \\
\hline 19 & 83.05 & $\mathrm{C}_{5} \mathrm{H}_{7} \mathrm{O}^{+}$ & valine \\
\hline 20 & 84.05 & $\mathrm{C}_{4} \mathrm{H}_{6} \mathrm{NO}^{+}$ & glutamine/glutamic acid \\
\hline 21 & 84.08 & $\mathrm{C}_{5} \mathrm{H}_{10} \mathrm{~N}^{+}$ & isoleucine/leucine/lysine \\
\hline 22 & 86.10 & $\mathrm{C}_{5} \mathrm{H}_{12} \mathrm{~N}^{+}$ & isoleucine/leucine \\
\hline 23 & 91.05 & $\mathrm{C}_{7} \mathrm{H}_{7}^{+}$ & phenylalanine/tyrosine \\
\hline 24 & 100.08 & $\mathrm{C}_{4} \mathrm{H}_{10} \mathrm{~N}_{3}{ }^{+}$ & arginine \\
\hline 25 & 102.06 & $\mathrm{C}_{4} \mathrm{H}_{8} \mathrm{NO}_{2}^{+}$ & glutamic acid/threonine \\
\hline 26 & 104.05 & $\mathrm{C}_{4} \mathrm{H}_{10} \mathrm{NS}^{+}$ & methionine \\
\hline 27 & 107.05 & $\mathrm{C}_{7} \mathrm{H}_{7} \mathrm{O}^{+}$ & tyrosine \\
\hline 28 & 110.07 & $\mathrm{C}_{5} \mathrm{H}_{8} \mathrm{~N}_{3}{ }^{+}$ & histidine \\
\hline 29 & 117.04 & $\mathrm{C}_{5} \mathrm{H}_{9} \mathrm{SO}^{+}$ & methionine \\
\hline 30 & 120.08 & $\mathrm{C}_{8} \mathrm{H}_{10} \mathrm{~N}^{+}$ & phenylalanine \\
\hline 31 & 130.07 & $\mathrm{C}_{9} \mathrm{H}_{8} \mathrm{~N}^{+}$ & tryptophan \\
\hline 32 & 131.05 & $\mathrm{C}_{9} \mathrm{H}_{7} \mathrm{O}^{+}$ & phenylalanine \\
\hline 33 & 136.07 & $\mathrm{C}_{8} \mathrm{H}_{10} \mathrm{NO}^{+}$ & tyrosine \\
\hline 34 & 147.04 & $\mathrm{C}_{9} \mathrm{H}_{7} \mathrm{O}_{2}^{+}$ & tyrosine \\
\hline 35 & 159.09 & $\mathrm{C}_{10} \mathrm{H}_{11} \mathrm{~N}_{2}^{+}$ & tryptophan \\
\hline
\end{tabular}
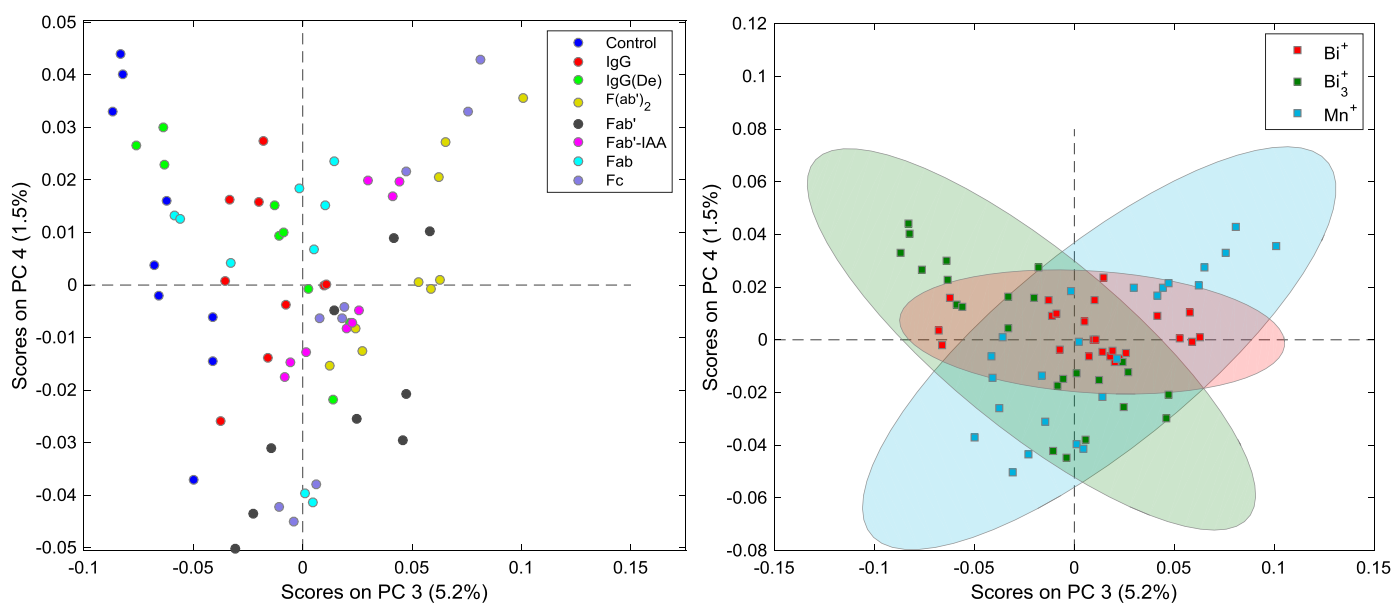

Figure S3. Principal component analysis of antibody fragments on silicon wafers PC 3 vs PC 4 as labeled by either protein fragment (left) or primary-ion source (right). ToF-SIMS peak list of 35 amino acid mass fragments was utilized. Ellipses show a $95 \%$ confidence level. 


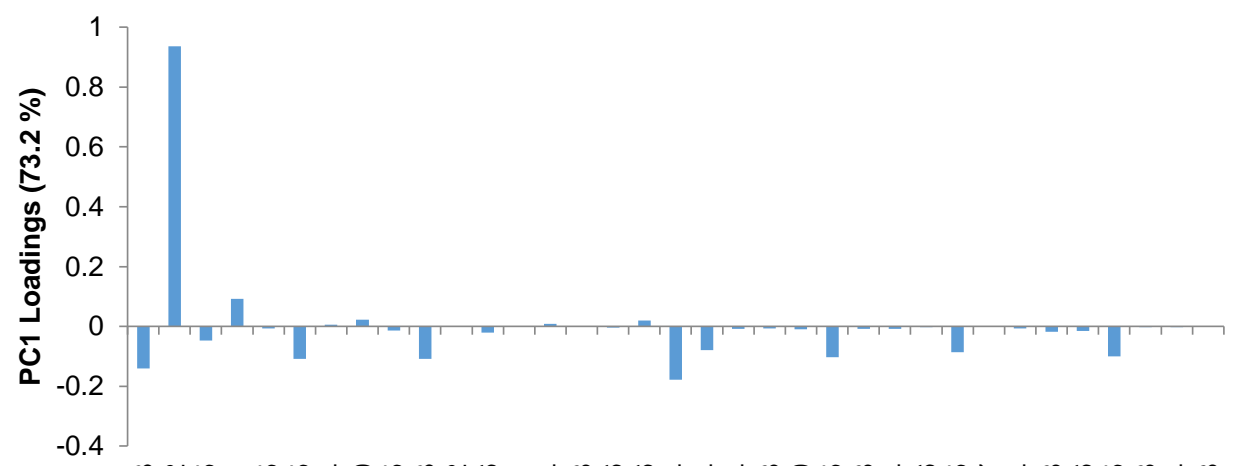

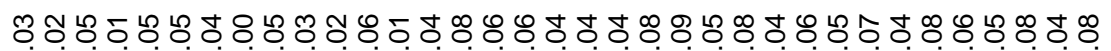

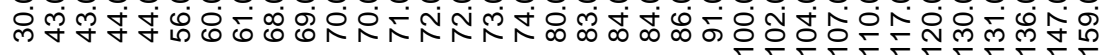
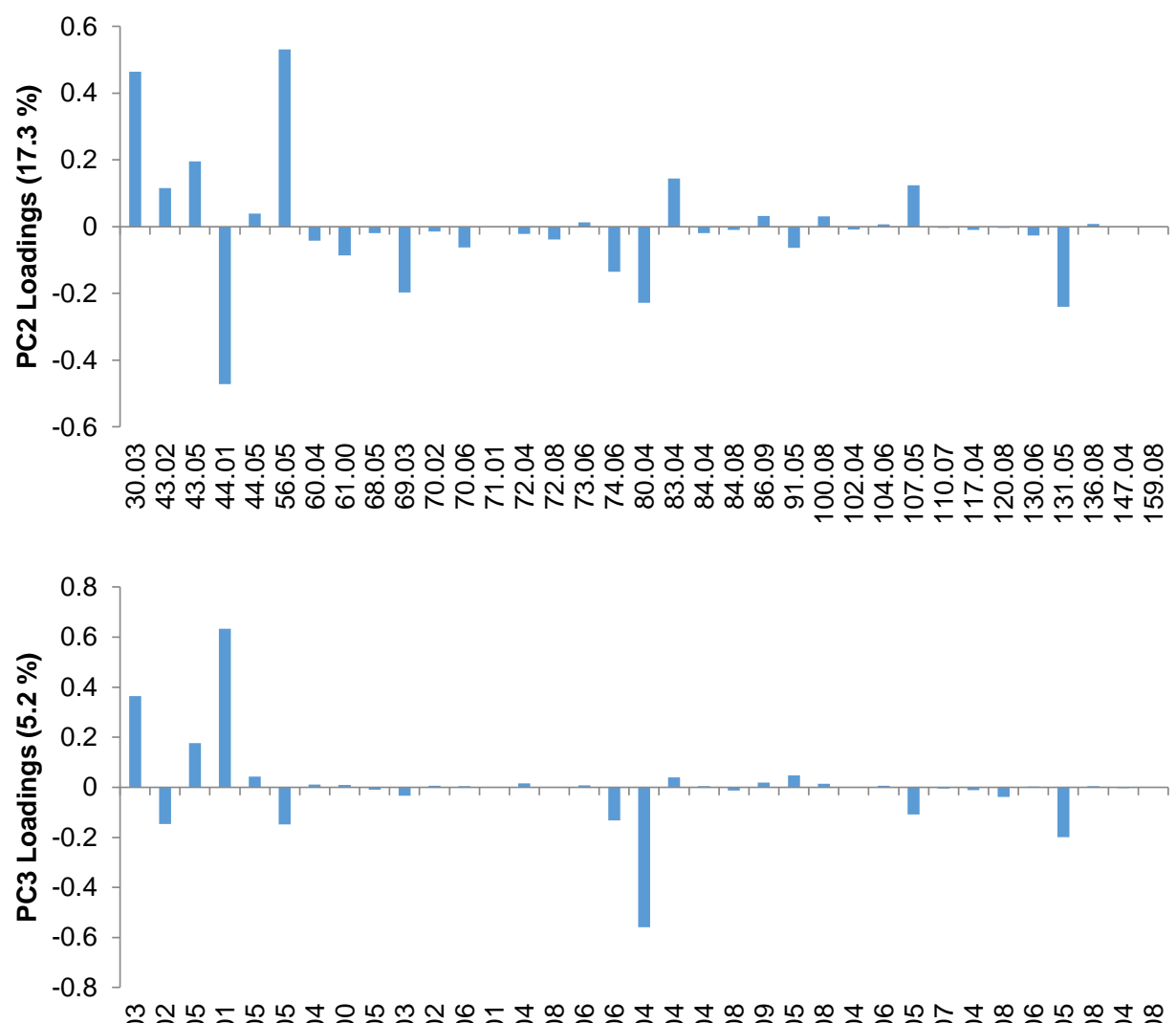

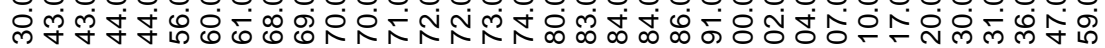

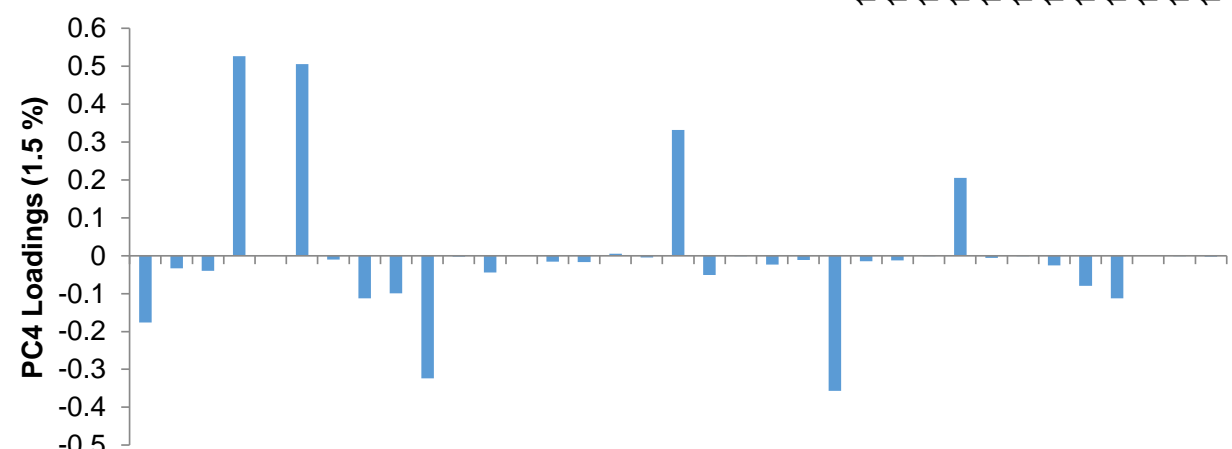

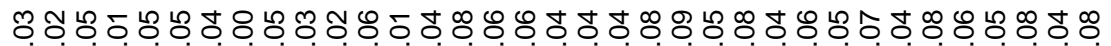

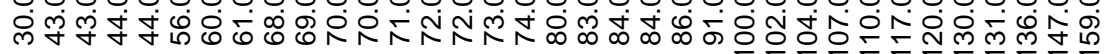

Fragment mass (m/z)

Figure S4. Loadings plots for PCA of all samples utilizing 35 amino acid mass fragments. 
Table S2. Sample list used in this work.

\begin{tabular}{|c|c|c|c|c|c|}
\hline $\begin{array}{l}\text { Sample } \\
\text { Number }\end{array}$ & Description & $\begin{array}{l}\text { Sample } \\
\text { Number }\end{array}$ & Description & $\begin{array}{l}\text { Sample } \\
\text { Number }\end{array}$ & Description \\
\hline 1 & $\mathrm{Bi}^{+}$Control Triplicate 1 & 25 & $\mathrm{Bi}_{3}{ }^{+}$Control Triplicate 1 & 49 & $\mathrm{Mn}^{+}$Control Triplicate 1 \\
\hline 2 & $\mathrm{Bi}^{+}$Control Triplicate 2 & 26 & $\mathrm{Bi}_{3}{ }^{+}$Control Triplicate 2 & 50 & $\mathrm{Mn}^{+}$Control Triplicate 2 \\
\hline 3 & $\mathrm{Bi}^{+}$Control Triplicate 3 & 27 & $\mathrm{Bi}_{3}{ }^{+}$Control Triplicate 3 & 51 & $\mathrm{Mn}^{+}$Control Triplicate 3 \\
\hline 4 & $\mathrm{Bi}^{+} \lg \mathrm{G}$ Triplicate 1 & 28 & $\mathrm{Bi}_{3}{ }^{+}$IgG Triplicate 1 & 52 & $\mathrm{Mn}^{+}$IgG Triplicate 1 \\
\hline 5 & $\mathrm{Bi}^{+}$IgG Triplicate 2 & 29 & $\mathrm{Bi}_{3}{ }^{+}$IgG Triplicate 2 & 53 & $\mathrm{Mn}^{+}$IgG Triplicate 2 \\
\hline 6 & $\mathrm{Bi}^{+}$IgG Triplicate 3 & 30 & $\mathrm{Bi}_{3}{ }^{+}$IgG Triplicate 3 & 54 & $\mathrm{Mn}^{+}$IgG Triplicate 3 \\
\hline 7 & $\mathrm{Bi}^{+} \lg \mathrm{G}(\mathrm{De})$ Triplicate 1 & 31 & $\mathrm{Bi}_{3}{ }^{+} \lg \mathrm{G}(\mathrm{De})$ Triplicate 1 & 55 & $\mathrm{Mn}^{+} \lg \mathrm{G}(\mathrm{De})$ Triplicate 1 \\
\hline 8 & $\mathrm{Bi}^{+} \operatorname{lgG}(\mathrm{De})$ Triplicate 2 & 32 & $\mathrm{Bi}_{3}{ }^{+} \lg \mathrm{G}(\mathrm{De})$ Triplicate 2 & 56 & $\mathrm{Mn}^{+} \operatorname{IgG}(\mathrm{De})$ Triplicate 2 \\
\hline 9 & $\mathrm{Bi}^{+} \lg \mathrm{G}(\mathrm{De})$ Triplicate 3 & 33 & $\mathrm{Bi}_{3}{ }^{+} \lg \mathrm{G}(\mathrm{De})$ Triplicate 3 & 57 & $\mathrm{Mn}^{+} \operatorname{IgG}(\mathrm{De})$ Triplicate 3 \\
\hline 10 & $\mathrm{Bi}^{+} \mathrm{F}\left(\mathrm{ab}^{\prime}\right)_{2}$ Triplicate 1 & 34 & $\mathrm{Bi}_{3}^{+} \mathrm{F}\left(\mathrm{ab}^{\prime}\right)_{2}$ Triplicate 1 & 58 & $\mathrm{Mn}^{+} \mathrm{F}\left(\mathrm{ab}^{\prime}\right)_{2}$ Triplicate 1 \\
\hline 11 & $\mathrm{Bi}^{+} \mathrm{F}\left(\mathrm{ab}^{\prime}\right)_{2}$ Triplicate 2 & 35 & $\mathrm{Bi}_{3}^{+} \mathrm{F}\left(\mathrm{ab}^{\prime}\right)_{2}$ Triplicate 2 & 59 & $\mathrm{Mn}^{+} \mathrm{F}\left(a b^{\prime}\right)_{2}$ Triplicate 2 \\
\hline 12 & $\mathrm{Bi}^{+} \mathrm{F}\left(\mathrm{ab}^{\prime}\right)_{2}$ Triplicate 3 & 36 & $\mathrm{Bi}_{3}{ }^{+} \mathrm{F}\left(\mathrm{ab}^{\prime}\right)_{2}$ Triplicate 3 & 60 & $\mathrm{Mn}^{+} \mathrm{F}\left(a b^{\prime}\right)_{2}$ Triplicate 3 \\
\hline 13 & $\mathrm{Bi}^{+} \mathrm{Fab}^{\prime}$ Triplicate 1 & 37 & $\mathrm{Bi}_{3}{ }^{+} \mathrm{Fab}^{\prime}$ Triplicate 1 & 61 & $\mathrm{Mn}^{+}$Fab' Triplicate 1 \\
\hline 14 & $\mathrm{Bi}^{+} \mathrm{Fab}^{\prime}$ Triplicate 2 & 38 & $\mathrm{Bi}_{3}{ }^{+} \mathrm{Fab}^{\prime}$ Triplicate 2 & 62 & $\mathrm{Mn}^{+}$Fab' Triplicate 2 \\
\hline 15 & $\mathrm{Bi}^{+} \mathrm{Fab}^{\prime}$ Triplicate 3 & 39 & $\mathrm{Bi}_{3}{ }^{+} \mathrm{Fab}^{\prime}$ Triplicate 3 & 63 & $\mathrm{Mn}^{+}$Fab' Triplicate 3 \\
\hline 16 & $\mathrm{Bi}^{+} \mathrm{Fab}^{\prime}-\mathrm{IAA}$ Triplicate 1 & 40 & $\mathrm{Bi}_{3}{ }^{+} \mathrm{Fab}^{\prime}-\mathrm{IAA}$ Triplicate 1 & 64 & $\mathrm{Mn}^{+} \mathrm{Fab}^{\prime}-\mathrm{IAA}$ Triplicate 1 \\
\hline 17 & $\mathrm{Bi}^{+} \mathrm{Fab}^{\prime}-\mathrm{IAA}$ Triplicate 2 & 41 & $\mathrm{Bi}_{3}{ }^{+} \mathrm{Fab}^{\prime}$-IAA Triplicate 2 & 65 & $\mathrm{Mn}^{+}$Fab'-IAA Triplicate 2 \\
\hline 18 & $\mathrm{Bi}^{+} \mathrm{Fab}^{\prime}-\mathrm{IAA}$ Triplicate 3 & 42 & $\mathrm{Bi}_{3}{ }^{+} \mathrm{Fab}^{\prime}$-IAA Triplicate 3 & 66 & $\mathrm{Mn}^{+}$Fab'-IAA Triplicate 3 \\
\hline 19 & $\mathrm{Bi}^{+}$Fab Triplicate 1 & 43 & $\mathrm{Bi}_{3}{ }^{+}$Fab Triplicate 1 & 67 & $\mathrm{Mn}^{+}$Fab Triplicate 1 \\
\hline 20 & $\mathrm{Bi}^{+}$Fab Triplicate 2 & 44 & $\mathrm{Bi}_{3}{ }^{+}$Fab Triplicate 2 & 68 & $\mathrm{Mn}^{+}$Fab Triplicate 2 \\
\hline 21 & $\mathrm{Bi}^{+}$Fab Triplicate 3 & 45 & $\mathrm{Bi}_{3}{ }^{+}$Fab Triplicate 3 & 69 & $\mathrm{Mn}^{+}$Fab Triplicate 3 \\
\hline 22 & $\mathrm{Bi}^{+} \mathrm{Fc}$ Triplicate 1 & 46 & $\mathrm{Bi}_{3}^{+} \mathrm{Fc}$ Triplicate 1 & 70 & $\mathrm{Mn}^{+}$Fc Triplicate 1 \\
\hline 23 & $\mathrm{Bi}^{+}$Fc Triplicate 2 & 47 & $\mathrm{Bi}_{3}{ }^{+}$Fc Triplicate 2 & 71 & $\mathrm{Mn}^{+}$Fc Triplicate 2 \\
\hline 24 & $\mathrm{Bi}^{+}$Fc Triplicate 3 & 48 & $\mathrm{Bi}_{3}{ }^{+} \mathrm{Fc}$ Triplicate 3 & 72 & $\mathrm{Mn}^{+}$Fc Triplicate 3 \\
\hline
\end{tabular}

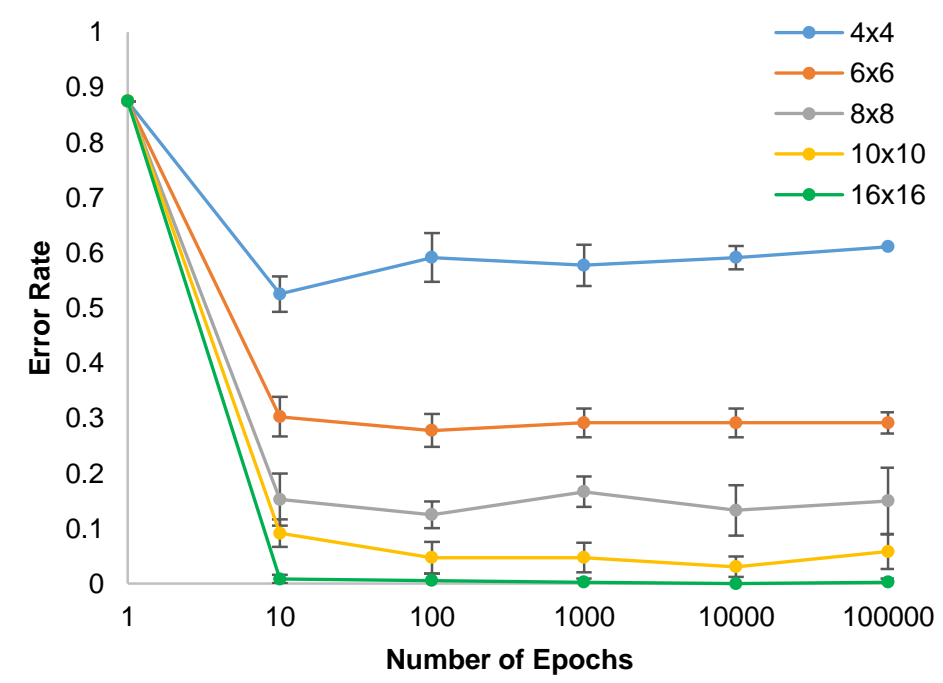

Figure S5. Error (Misclassification) rate as function of number of epochs for the different map sizes including those identified in Figure 11. Points are average of 5 individual map reconstructions. Error bars \pm standard deviation. 


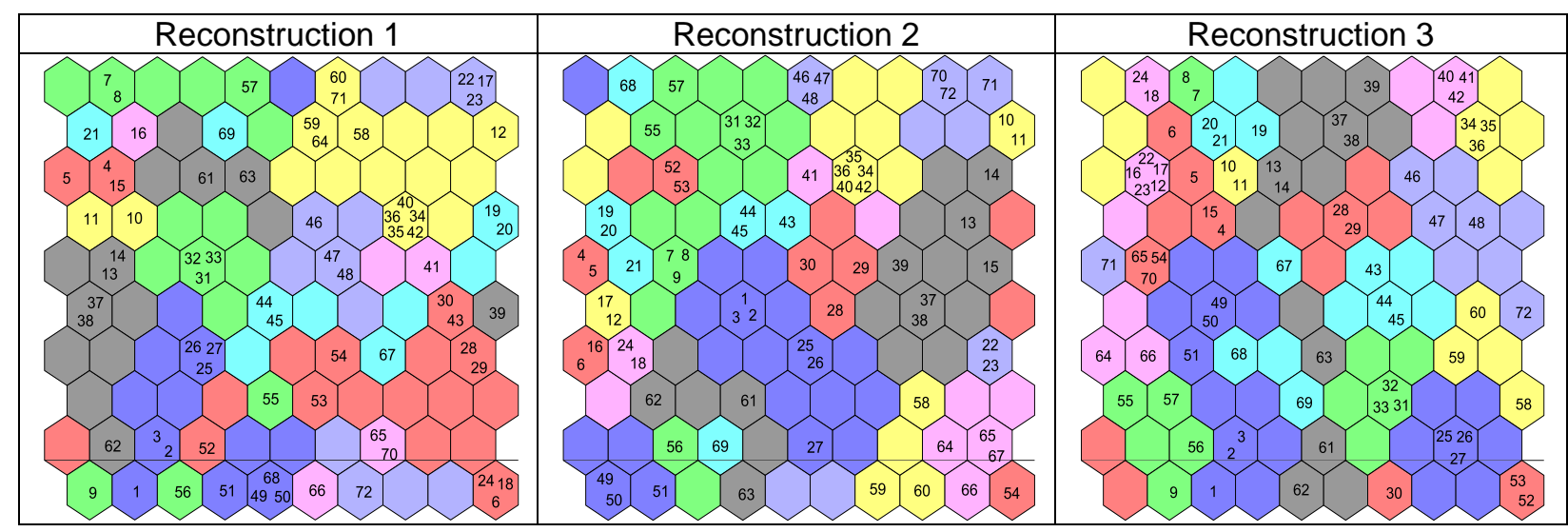

Figure S6. Three separate CP-ANNs constructed using identical parameters; $10 \times 10$ NN with 100000 epochs. Error (misclassification) rates are $15.3 \%, 8.3 \%$, and $9.7 \%$ for reconstructions 1, 2, and 3 respectively. Eight classes: Control wafer, dark purple; whole antibody lgG, red; deglycosylated antibody IgG(De), light green; $F\left(a b^{\prime}\right)_{2}$, yellow; Fab', gray; iodoacetamide blocked Fab', Fab'-IAA, pink; Fab, light blue; Fc, light purple. ToF-SIMS peak list of 35 amino acid mass fragments was utilized. Numbers represent individual samples; full details in Table S2. 
Table S3. Correlation of the User-Defined Class (Input) with the ANN Assigned Class (Output) for Reconstruction 1 (from Figure 12) Sorted by Primary-Ion Source: (a) Total Summed Average (AVG), (b) $\mathrm{Mn}^{+}$, (c) $\mathrm{Bi}^{+}$, and (d) $\mathrm{Bi}_{3}{ }^{+}$.

\begin{tabular}{|l|c|c|c|c|c|c|c|c|}
\hline a) AVG & \multicolumn{9}{|c|}{ User-defined Class } \\
\hline ANN Assigned & Control & IgG & IgG(De) & F(ab' $)_{2}$ & Fab' & Fab'-IAA & Fab & Fc \\
\hline Control & 1.00 & 0.00 & 0.00 & 0.00 & 0.00 & 0.00 & 0.00 & 0.00 \\
\hline $\operatorname{lgG}$ & 0.00 & 1.00 & 0.00 & 0.00 & 0.00 & 0.00 & 0.00 & 0.00 \\
\hline $\operatorname{lgG}(\mathrm{De})$ & 0.00 & 0.00 & 1.00 & 0.00 & 0.00 & 0.00 & 0.00 & 0.00 \\
\hline $\mathrm{F}\left(\mathrm{ab}^{\prime}\right)_{2}$ & 0.00 & 0.00 & 0.00 & 0.78 & 0.00 & 0.22 & 0.00 & 0.00 \\
\hline Fab' & 0.00 & 0.00 & 0.00 & 0.00 & 1.00 & 0.00 & 0.00 & 0.00 \\
\hline Fab'-IAA & 0.00 & 0.00 & 0.00 & 0.22 & 0.00 & 0.78 & 0.00 & 0.00 \\
\hline Fab & 0.00 & 0.00 & 0.00 & 0.00 & 0.00 & 0.00 & 1.00 & 0.00 \\
\hline Fc & 0.00 & 0.00 & 0.00 & 0.00 & 0.00 & 0.00 & 0.00 & 1.00 \\
\hline
\end{tabular}

\begin{tabular}{|l|c|c|c|c|c|c|c|c|}
\hline b) $\mathrm{Mn}^{+}$ & \multicolumn{9}{|c|}{ User-defined Class } \\
\hline ANN Assigned & Control & IgG & IgG(De) & F $\left(\mathrm{ab}^{\prime}\right)_{2}$ & Fab' & Fab'-IAA & Fab & Fc \\
\hline Control & 1.00 & 0.00 & 0.00 & 0.00 & 0.00 & 0.00 & 0.00 & 0.00 \\
\hline IgG & 0.00 & 1.00 & 0.00 & 0.00 & 0.00 & 0.00 & 0.00 & 0.00 \\
\hline $\mathrm{IgG}(\mathrm{De})$ & 0.00 & 0.00 & 1.00 & 0.00 & 0.00 & 0.00 & 0.00 & 0.00 \\
\hline $\mathrm{F}\left(\mathrm{ab}^{\prime}\right)_{2}$ & 0.00 & 0.00 & 0.00 & 0.50 & 0.00 & 0.50 & 0.00 & 0.00 \\
\hline $\mathrm{Fab}^{\prime}$ & 0.00 & 0.00 & 0.00 & 0.00 & 1.00 & 0.00 & 0.00 & 0.00 \\
\hline Fab'-IAA & 0.00 & 0.00 & 0.00 & 0.50 & 0.00 & 0.50 & 0.00 & 0.00 \\
\hline Fab & 0.00 & 0.00 & 0.00 & 0.00 & 0.00 & 0.00 & 1.00 & 0.00 \\
\hline Fc & 0.00 & 0.00 & 0.00 & 0.00 & 0.00 & 0.00 & 0.00 & 1.00 \\
\hline
\end{tabular}

\begin{tabular}{|l|c|c|c|c|c|c|c|c|}
\hline${\text { c) } \mathrm{Bi}^{+}}^{+}$ & \multicolumn{9}{|c|}{ User-defined Class } \\
\hline ANN Assigned & Control & IgG & IgG(De) & F $\left(\mathrm{ab}^{\prime}\right)_{2}$ & Fab' & Fab'-IAA & Fab & Fc \\
\hline Control & 1.00 & 0.00 & 0.00 & 0.00 & 0.00 & 0.00 & 0.00 & 0.00 \\
\hline IgG & 0.00 & 1.00 & 0.00 & 0.00 & 0.00 & 0.00 & 0.00 & 0.00 \\
\hline $\operatorname{lgG}(\mathrm{De})$ & 0.00 & 0.00 & 1.00 & 0.00 & 0.00 & 0.00 & 0.00 & 0.00 \\
\hline $\mathrm{F}\left(\mathrm{ab}^{\prime}\right)_{2}$ & 0.00 & 0.00 & 0.00 & 0.83 & 0.00 & 0.17 & 0.00 & 0.00 \\
\hline $\mathrm{Fab}^{\prime}$ & 0.00 & 0.00 & 0.00 & 0.00 & 1.00 & 0.00 & 0.00 & 0.00 \\
\hline Fab'-IAA & 0.00 & 0.00 & 0.00 & 0.17 & 0.00 & 0.83 & 0.00 & 0.00 \\
\hline Fab & 0.00 & 0.00 & 0.00 & 0.00 & 0.00 & 0.00 & 1.00 & 0.00 \\
\hline Fc & 0.00 & 0.00 & 0.00 & 0.00 & 0.00 & 0.00 & 0.00 & 1.00 \\
\hline
\end{tabular}

\begin{tabular}{|c|c|c|c|c|c|c|c|c|}
\hline d) $\mathrm{Bi}_{3}{ }^{+}$ & \multicolumn{8}{|c|}{ User-defined Class } \\
\hline ANN Assigned & Control & $\lg G$ & $\lg G(D e)$ & $F\left(a b^{\prime}\right)_{2}$ & Fab' & Fab'-IAA & $\mathrm{Fab}$ & Fc \\
\hline Control & 1.00 & 0.00 & 0.00 & 0.00 & 0.00 & 0.00 & 0.00 & 0.00 \\
\hline $\lg G$ & 0.00 & 1.00 & 0.00 & 0.00 & 0.00 & 0.00 & 0.00 & 0.00 \\
\hline $\lg G(D e)$ & 0.00 & 0.00 & 1.00 & 0.00 & 0.00 & 0.00 & 0.00 & 0.00 \\
\hline$F\left(a b^{\prime}\right)_{2}$ & 0.00 & 0.00 & 0.00 & 1.00 & 0.00 & 0.00 & 0.00 & 0.00 \\
\hline Fab' & 0.00 & 0.00 & 0.00 & 0.00 & 1.00 & 0.00 & 0.00 & 0.00 \\
\hline Fab'-IAA & 0.00 & 0.00 & 0.00 & 0.00 & 0.00 & 1.00 & 0.00 & 0.00 \\
\hline Fab & 0.00 & 0.00 & 0.00 & 0.00 & 0.00 & 0.00 & 1.00 & 0.00 \\
\hline $\mathrm{Fc}$ & 0.00 & 0.00 & 0.00 & 0.00 & 0.00 & 0.00 & 0.00 & 1.00 \\
\hline
\end{tabular}

Table S4. Correlation of the User-Defined Class (Input) with the ANN Assigned Class (Output) for Reconstruction 2 (from Figure 12) Sorted by Primary-Ion Source: (a) Total Summed Average (AVG), (b) $\mathrm{Mn}^{+}$, (c) $\mathrm{Bi}^{+}$, and (d) $\mathrm{Bi}_{3}{ }^{+}$. 


\begin{tabular}{|l|c|c|c|c|c|c|c|c|}
\hline a) AVG & \multicolumn{8}{|c|}{ User-defined Class } \\
\hline ANN Assigned & Control & $\operatorname{lgG}$ & $\lg G(\mathrm{De})$ & $\mathrm{F}\left(\mathrm{ab}^{\prime}\right)_{2}$ & $\mathrm{Fab}^{\prime}$ & Fab'-IAA & Fab & Fc \\
\hline Control & 0.92 & 0.00 & 0.08 & 0.00 & 0.00 & 0.00 & 0.00 & 0.00 \\
\hline $\operatorname{lgG}$ & 0.00 & 0.93 & 0.00 & 0.00 & 0.00 & 0.00 & 0.07 & 0.00 \\
\hline $\operatorname{lgG}(\mathrm{De})$ & 0.08 & 0.00 & 0.86 & 0.00 & 0.00 & 0.00 & 0.06 & 0.00 \\
\hline F(ab') & 0.00 & 0.00 & 0.00 & 0.78 & 0.00 & 0.22 & 0.00 & 0.00 \\
\hline Fab' & 0.00 & 0.00 & 0.00 & 0.00 & 1.00 & 0.00 & 0.00 & 0.00 \\
\hline Fab'-IAA & 0.00 & 0.00 & 0.00 & 0.22 & 0.00 & 0.78 & 0.00 & 0.00 \\
\hline Fab & 0.00 & 0.07 & 0.06 & 0.00 & 0.00 & 0.00 & 0.87 & 0.00 \\
\hline Fc & 0.00 & 0.00 & 0.00 & 0.00 & 0.00 & 0.00 & 0.00 & 1.00 \\
\hline
\end{tabular}

\begin{tabular}{|c|c|c|c|c|c|c|c|c|}
\hline b) $\mathrm{Mn}^{+}$ & \multicolumn{8}{|c|}{ User-defined Class } \\
\hline ANN Assigned & Control & $\lg G$ & $\lg G(\mathrm{De})$ & $F\left(a b^{\prime}\right)_{2}$ & Fab' & Fab'-IAA & Fab & Fc \\
\hline Control & 0.75 & 0.00 & 0.25 & 0.00 & 0.00 & 0.00 & 0.00 & 0.00 \\
\hline $\lg G$ & 0.00 & 0.78 & 0.00 & 0.00 & 0.00 & 0.00 & 0.22 & 0.00 \\
\hline $\lg G(D e)$ & 0.25 & 0.00 & 0.58 & 0.00 & 0.00 & 0.00 & 0.17 & 0.00 \\
\hline$F\left(a b^{\prime}\right)_{2}$ & 0.00 & 0.00 & 0.00 & 0.50 & 0.00 & 0.50 & 0.00 & 0.00 \\
\hline Fab' & 0.00 & 0.00 & 0.00 & 0.00 & 1.00 & 0.00 & 0.00 & 0.00 \\
\hline Fab'-IAA & 0.00 & 0.00 & 0.00 & 0.50 & 0.00 & 0.50 & 0.00 & 0.00 \\
\hline Fab & 0.00 & 0.22 & 0.17 & 0.00 & 0.00 & 0.00 & 0.61 & 0.00 \\
\hline Fc & 0.00 & 0.00 & 0.00 & 0.00 & 0.00 & 0.00 & 0.00 & 1.00 \\
\hline
\end{tabular}

\begin{tabular}{|c|c|c|c|c|c|c|c|c|}
\hline c) $\mathrm{Bi}^{+}$ & \multicolumn{8}{|c|}{ User-defined Class } \\
\hline ANN Assigned & Control & $\lg G$ & $\lg G(\mathrm{De})$ & $F\left(a b^{\prime}\right)_{2}$ & $\mathrm{Fab}^{\prime}$ & Fab'-IAA & Fab & Fc \\
\hline Control & 1.00 & 0.00 & 0.00 & 0.00 & 0.00 & 0.00 & 0.00 & 0.00 \\
\hline $\lg G$ & 0.00 & 1.00 & 0.00 & 0.00 & 0.00 & 0.00 & 0.00 & 0.00 \\
\hline $\lg G(D e)$ & 0.00 & 0.00 & 1.00 & 0.00 & 0.00 & 0.00 & 0.00 & 0.00 \\
\hline$F\left(a b^{\prime}\right)_{2}$ & 0.00 & 0.00 & 0.00 & 0.83 & 0.00 & 0.17 & 0.00 & 0.00 \\
\hline Fab' & 0.00 & 0.00 & 0.00 & 0.00 & 1.00 & 0.00 & 0.00 & 0.00 \\
\hline Fab'-IAA & 0.00 & 0.00 & 0.00 & 0.17 & 0.00 & 0.83 & 0.00 & 0.00 \\
\hline Fab & 0.00 & 0.00 & 0.00 & 0.00 & 0.00 & 0.00 & 1.00 & 0.00 \\
\hline Fc & 0.00 & 0.00 & 0.00 & 0.00 & 0.00 & 0.00 & 0.00 & 1.00 \\
\hline
\end{tabular}

\begin{tabular}{|c|c|c|c|c|c|c|c|c|}
\hline d) $\mathrm{Bi}_{3}^{+}$ & \multicolumn{8}{|c|}{ User-defined Class } \\
\hline ANN Assigned & Control & $\lg G$ & $\lg G(D e)$ & $\mathrm{F}\left(a b^{\prime}\right)_{2}$ & Fab' & Fab'-IAA & Fab & Fc \\
\hline Control & 1.00 & 0.00 & 0.00 & 0.00 & 0.00 & 0.00 & 0.00 & 0.00 \\
\hline $\lg G$ & 0.00 & 1.00 & 0.00 & 0.00 & 0.00 & 0.00 & 0.00 & 0.00 \\
\hline $\lg G(D e)$ & 0.00 & 0.00 & 1.00 & 0.00 & 0.00 & 0.00 & 0.00 & 0.00 \\
\hline$F\left(a b^{\prime}\right)_{2}$ & 0.00 & 0.00 & 0.00 & 1.00 & 0.00 & 0.00 & 0.00 & 0.00 \\
\hline Fab' & 0.00 & 0.00 & 0.00 & 0.00 & 1.00 & 0.00 & 0.00 & 0.00 \\
\hline Fab'-IAA & 0.00 & 0.00 & 0.00 & 0.00 & 0.00 & 1.00 & 0.00 & 0.00 \\
\hline Fab & 0.00 & 0.00 & 0.00 & 0.00 & 0.00 & 0.00 & 1.00 & 0.00 \\
\hline Fc & 0.00 & 0.00 & 0.00 & 0.00 & 0.00 & 0.00 & 0.00 & 1.00 \\
\hline
\end{tabular}

Table S5. Correlation of the User-Defined Class (Input) with the ANN Assigned Class (Output) for Reconstruction 3 (from Figure 12) Sorted by Primary-lon Source: (a) Total Summed Average (AVG), (b) $\mathrm{Mn}^{+}$, (c) $\mathrm{Bi}^{+}$, and (d) $\mathrm{Bi}_{3}{ }^{+}$.

\begin{tabular}{|l|l}
\hline a) AVG & User-defined Class \\
\hline
\end{tabular}




\begin{tabular}{|l|c|c|c|c|c|c|c|c|} 
ANN Assigned & Control & $\lg G$ & $\lg G(D e)$ & $F\left(a b^{\prime}\right)_{2}$ & $F a b^{\prime}$ & $F a b^{\prime}-I A A$ & $F a b$ & $F c$ \\
\hline Control & 1.00 & 0.00 & 0.00 & 0.00 & 0.00 & 0.00 & 0.00 & 0.00 \\
\hline $\operatorname{lgG}$ & 0.00 & 0.94 & 0.06 & 0.00 & 0.00 & 0.00 & 0.00 & 0.00 \\
\hline $\lg G(D e)$ & 0.00 & 0.06 & 0.94 & 0.00 & 0.00 & 0.00 & 0.00 & 0.00 \\
\hline$F\left(a b^{\prime}\right)_{2}$ & 0.00 & 0.00 & 0.00 & 1.00 & 0.00 & 0.00 & 0.00 & 0.00 \\
\hline Fab' $^{\prime}$ & 0.00 & 0.00 & 0.00 & 0.00 & 1.00 & 0.00 & 0.00 & 0.00 \\
\hline Fab'-IAA & 0.00 & 0.00 & 0.00 & 0.00 & 0.00 & 0.94 & 0.06 & 0.00 \\
\hline Fab & 0.00 & 0.00 & 0.00 & 0.00 & 0.00 & 0.06 & 0.94 & 0.00 \\
\hline Fc & 0.00 & 0.00 & 0.00 & 0.00 & 0.00 & 0.00 & 0.00 & 1.00 \\
\hline
\end{tabular}

\begin{tabular}{|l|c|c|c|c|c|c|c|c|}
\hline \hline b) $\mathrm{Mn}^{+}$ & \multicolumn{9}{|c|}{ User-defined Class } \\
\hline ANN Assigned & Control & IgG & IgG(De) & F(ab' $)_{2}$ & Fab' & Fab'-IAA & Fab & Fc \\
\hline Control & 1.00 & 0.00 & 0.00 & 0.00 & 0.00 & 0.00 & 0.00 & 0.00 \\
\hline IgG & 0.00 & 0.83 & 0.17 & 0.00 & 0.00 & 0.00 & 0.00 & 0.00 \\
\hline $\operatorname{lgG}(\mathrm{De})$ & 0.00 & 0.17 & 0.83 & 0.00 & 0.00 & 0.00 & 0.00 & 0.00 \\
\hline $\mathrm{F}\left(\mathrm{ab}^{\prime}\right)_{2}$ & 0.00 & 0.00 & 0.00 & 1.00 & 0.00 & 0.00 & 0.00 & 0.00 \\
\hline $\mathrm{Fab}^{\prime}$ & 0.00 & 0.00 & 0.00 & 0.00 & 1.00 & 0.00 & 0.00 & 0.00 \\
\hline Fab'-IAA & 0.00 & 0.00 & 0.00 & 0.00 & 0.00 & 0.83 & 0.17 & 0.00 \\
\hline Fab & 0.00 & 0.00 & 0.00 & 0.00 & 0.00 & 0.17 & 0.83 & 0.00 \\
\hline Fc & 0.00 & 0.00 & 0.00 & 0.00 & 0.00 & 0.00 & 0.00 & 1.00 \\
\hline
\end{tabular}

\begin{tabular}{|c|c|c|c|c|c|c|c|c|}
\hline c) $\mathrm{Bi}^{+}$ & \multicolumn{8}{|c|}{ User-defined Class } \\
\hline ANN Assigned & Control & $\lg G$ & $\lg G(D e)$ & $\mathrm{F}\left(a b^{\prime}\right)_{2}$ & $F a^{\prime}$ & Fab'-IAA & Fab & Fc \\
\hline Control & 1.00 & 0.00 & 0.00 & 0.00 & 0.00 & 0.00 & 0.00 & 0.00 \\
\hline $\lg G$ & 0.00 & 1.00 & 0.00 & 0.00 & 0.00 & 0.00 & 0.00 & 0.00 \\
\hline $\lg G(D e)$ & 0.00 & 0.00 & 1.00 & 0.00 & 0.00 & 0.00 & 0.00 & 0.00 \\
\hline$F\left(a b^{\prime}\right)_{2}$ & 0.00 & 0.00 & 0.00 & 1.00 & 0.00 & 0.00 & 0.00 & 0.00 \\
\hline Fab' & 0.00 & 0.00 & 0.00 & 0.00 & 1.00 & 0.00 & 0.00 & 0.00 \\
\hline Fab'-IAA & 0.00 & 0.00 & 0.00 & 0.00 & 0.00 & 1.00 & 0.00 & 0.00 \\
\hline Fab & 0.00 & 0.00 & 0.00 & 0.00 & 0.00 & 0.00 & 1.00 & 0.00 \\
\hline Fc & 0.00 & 0.00 & 0.00 & 0.00 & 0.00 & 0.00 & 0.00 & 1.00 \\
\hline
\end{tabular}

\begin{tabular}{|c|c|c|c|c|c|c|c|c|}
\hline d) $\mathrm{Bi}_{3}{ }^{+}$ & \multicolumn{8}{|c|}{ User-defined Class } \\
\hline ANN Assigned & Control & $\lg G$ & $\lg G(D e)$ & $\mathrm{F}\left(a b^{\prime}\right)_{2}$ & Fab' & Fab'-IAA & Fab & Fc \\
\hline Control & 1.00 & 0.00 & 0.00 & 0.00 & 0.00 & 0.00 & 0.00 & 0.00 \\
\hline $\lg G$ & 0.00 & 1.00 & 0.00 & 0.00 & 0.00 & 0.00 & 0.00 & 0.00 \\
\hline $\lg G(D e)$ & 0.00 & 0.00 & 1.00 & 0.00 & 0.00 & 0.00 & 0.00 & 0.00 \\
\hline$F\left(a b^{\prime}\right)_{2}$ & 0.00 & 0.00 & 0.00 & 1.00 & 0.00 & 0.00 & 0.00 & 0.00 \\
\hline$F a b^{\prime}$ & 0.00 & 0.00 & 0.00 & 0.00 & 1.00 & 0.00 & 0.00 & 0.00 \\
\hline Fab'-IAA & 0.00 & 0.00 & 0.00 & 0.00 & 0.00 & 1.00 & 0.00 & 0.00 \\
\hline $\mathrm{Fab}$ & 0.00 & 0.00 & 0.00 & 0.00 & 0.00 & 0.00 & 1.00 & 0.00 \\
\hline Fc & 0.00 & 0.00 & 0.00 & 0.00 & 0.00 & 0.00 & 0.00 & 1.00 \\
\hline
\end{tabular}

Table S6. Correlation of the User-Defined Class (Input) with the ANN Assigned Class (Output) Averaged across the Three Reconstructions (from Figure S6) and Sorted by Primary-lon Source: (a) Total Summed Average (AVG), (b) $\mathrm{Mn}^{+}$, (c) $\mathrm{Bi}^{+}$, and (d) $\mathrm{Bi}_{3}{ }^{+}$. ToF-SIMS peak list of 35 amino acid mass fragments was utilized.

\begin{tabular}{|l|c|c|c|c|c|c|c|c|}
\hline a) AVG & \multicolumn{7}{|c|}{ User-defined Class } \\
\hline ANN Assigned & Control & $\lg G$ & $\lg G(\mathrm{De})$ & $\mathrm{F}\left(\mathrm{ab}^{\prime}\right)_{2}$ & $\mathrm{Fab}^{\prime}$ & $\mathrm{Fab}^{\prime}-\mathrm{IAA}$ & $\mathrm{Fab}$ & $\mathrm{Fc}$ \\
\hline
\end{tabular}




\begin{tabular}{|l|l|l|l|l|l|l|l|l|}
\cline { 2 - 8 } Control & 0.94 & 0.04 & 0.00 & 0.00 & 0.00 & 0.00 & 0.02 & 0.00 \\
\hline $\operatorname{lgG}$ & 0.00 & 0.85 & 0.04 & 0.00 & 0.04 & 0.04 & 0.02 & 0.02 \\
\hline $\operatorname{lgG}(\mathrm{De})$ & 0.00 & 0.00 & 0.96 & 0.04 & 0.00 & 0.00 & 0.00 & 0.00 \\
\hline $\mathrm{F}\left(\mathrm{ab}^{\prime}\right)_{2}$ & 0.00 & 0.00 & 0.00 & 0.80 & 0.04 & 0.14 & 0.00 & 0.03 \\
\hline $\mathrm{Fab}^{\prime}$ & 0.00 & 0.04 & 0.00 & 0.02 & 0.93 & 0.02 & 0.00 & 0.00 \\
\hline $\mathrm{Fab}$ '-IAA & 0.00 & 0.04 & 0.00 & 0.14 & 0.00 & 0.64 & 0.05 & 0.13 \\
\hline $\mathrm{Fab}$ & 0.02 & 0.02 & 0.00 & 0.00 & 0.00 & 0.04 & 0.90 & 0.02 \\
\hline Fc & 0.00 & 0.02 & 0.00 & 0.03 & 0.00 & 0.13 & 0.00 & 0.81 \\
\hline
\end{tabular}

\begin{tabular}{|l|c|c|c|c|c|c|c|c|}
\hline b) $\mathrm{Mn}^{+}$ & \multicolumn{7}{|c|}{ User-defined Class } \\
\hline ANN Assigned & Control & IgG & IgG(De) & $F\left(a^{\prime}\right)_{2}$ & Fab' & Fab'-IAA & Fab & Fc \\
\hline Control & 0.83 & 0.10 & 0.00 & 0.00 & 0.00 & 0.00 & 0.07 & 0.00 \\
\hline $\operatorname{lgG}$ & 0.00 & 0.93 & 0.00 & 0.00 & 0.00 & 0.04 & 0.00 & 0.04 \\
\hline $\operatorname{lgG}(\mathrm{De})$ & 0.00 & 0.00 & 1.00 & 0.00 & 0.00 & 0.00 & 0.00 & 0.00 \\
\hline $\mathrm{F}\left(\mathrm{ab}^{\prime}\right)_{2}$ & 0.00 & 0.00 & 0.00 & 0.89 & 0.00 & 0.06 & 0.00 & 0.06 \\
\hline $\mathrm{Fab}^{\prime}$ & 0.00 & 0.00 & 0.00 & 0.00 & 1.00 & 0.00 & 0.00 & 0.00 \\
\hline Fab'-IAA & 0.00 & 0.04 & 0.00 & 0.06 & 0.00 & 0.76 & 0.06 & 0.09 \\
\hline Fab & 0.22 & 0.00 & 0.00 & 0.00 & 0.00 & 0.00 & 0.78 & 0.00 \\
\hline Fc & 0.00 & 0.04 & 0.00 & 0.06 & 0.00 & 0.09 & 0.00 & 0.81 \\
\hline
\end{tabular}

\begin{tabular}{|c|c|c|c|c|c|c|c|c|}
\hline c) $\mathrm{Bi}^{+}$ & \multicolumn{8}{|c|}{ User-defined Class } \\
\hline ANN Assigned & Control & $\lg G$ & $\lg G(D e)$ & $F\left(a b^{\prime}\right)_{2}$ & $\mathrm{Fab}^{\prime}$ & 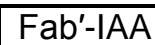 & Fab & Fc \\
\hline Control & 1.00 & 0.00 & 0.00 & 0.00 & 0.00 & 0.00 & 0.00 & 0.00 \\
\hline $\lg G$ & 0.00 & 0.76 & 0.00 & 0.00 & 0.11 & 0.09 & 0.00 & 0.04 \\
\hline $\lg G(D e)$ & 0.00 & 0.00 & 1.00 & 0.00 & 0.00 & 0.00 & 0.00 & 0.00 \\
\hline$F\left(a b^{\prime}\right)_{2}$ & 0.00 & 0.00 & 0.00 & 0.86 & 0.00 & 0.10 & 0.00 & 0.04 \\
\hline Fab' & 0.00 & 0.11 & 0.00 & 0.00 & 0.89 & 0.00 & 0.00 & 0.00 \\
\hline Fab'-IAA & 0.00 & 0.09 & 0.00 & 0.10 & 0.00 & 0.50 & 0.00 & 0.31 \\
\hline Fab & 0.00 & 0.00 & 0.00 & 0.00 & 0.00 & 0.00 & 1.00 & 0.00 \\
\hline Fc & 0.00 & 0.04 & 0.00 & 0.04 & 0.00 & 0.31 & 0.00 & 0.61 \\
\hline
\end{tabular}

\begin{tabular}{|c|c|c|c|c|c|c|c|c|}
\hline d) $\mathrm{Bi}_{3}{ }^{+}$ & \multicolumn{8}{|c|}{ User-defined Class } \\
\hline ANN Assigned & Control & $\lg G$ & $\lg G(\mathrm{De})$ & $F\left(a b^{\prime}\right)_{2}$ & $\mathrm{Fab}^{\prime}$ & Fab'-IAA & $\mathrm{Fab}$ & Fc \\
\hline Control & 1.00 & 0.00 & 0.00 & 0.00 & 0.00 & 0.00 & 0.00 & 0.00 \\
\hline $\lg G$ & 0.00 & 0.94 & 0.00 & 0.00 & 0.00 & 0.00 & 0.06 & 0.00 \\
\hline $\lg G(D e)$ & 0.00 & 0.00 & 1.00 & 0.00 & 0.00 & 0.00 & 0.00 & 0.00 \\
\hline$F\left(a b^{\prime}\right)_{2}$ & 0.00 & 0.00 & 0.00 & 0.73 & 0.00 & 0.27 & 0.00 & 0.00 \\
\hline Fab' & 0.00 & 0.00 & 0.00 & 0.00 & 1.00 & 0.00 & 0.00 & 0.00 \\
\hline Fab'-IAA & 0.00 & 0.00 & 0.00 & 0.27 & 0.00 & 0.73 & 0.00 & 0.00 \\
\hline $\mathrm{Fab}$ & 0.00 & 0.06 & 0.00 & 0.00 & 0.00 & 0.00 & 0.94 & 0.00 \\
\hline Fc & 0.00 & 0.00 & 0.00 & 0.00 & 0.00 & 0.00 & 0.00 & 1.00 \\
\hline
\end{tabular}

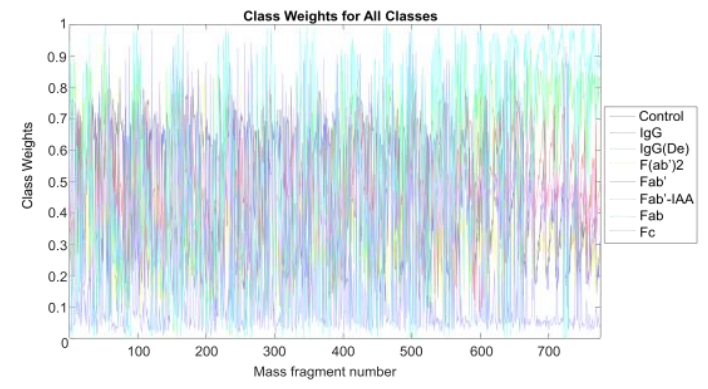



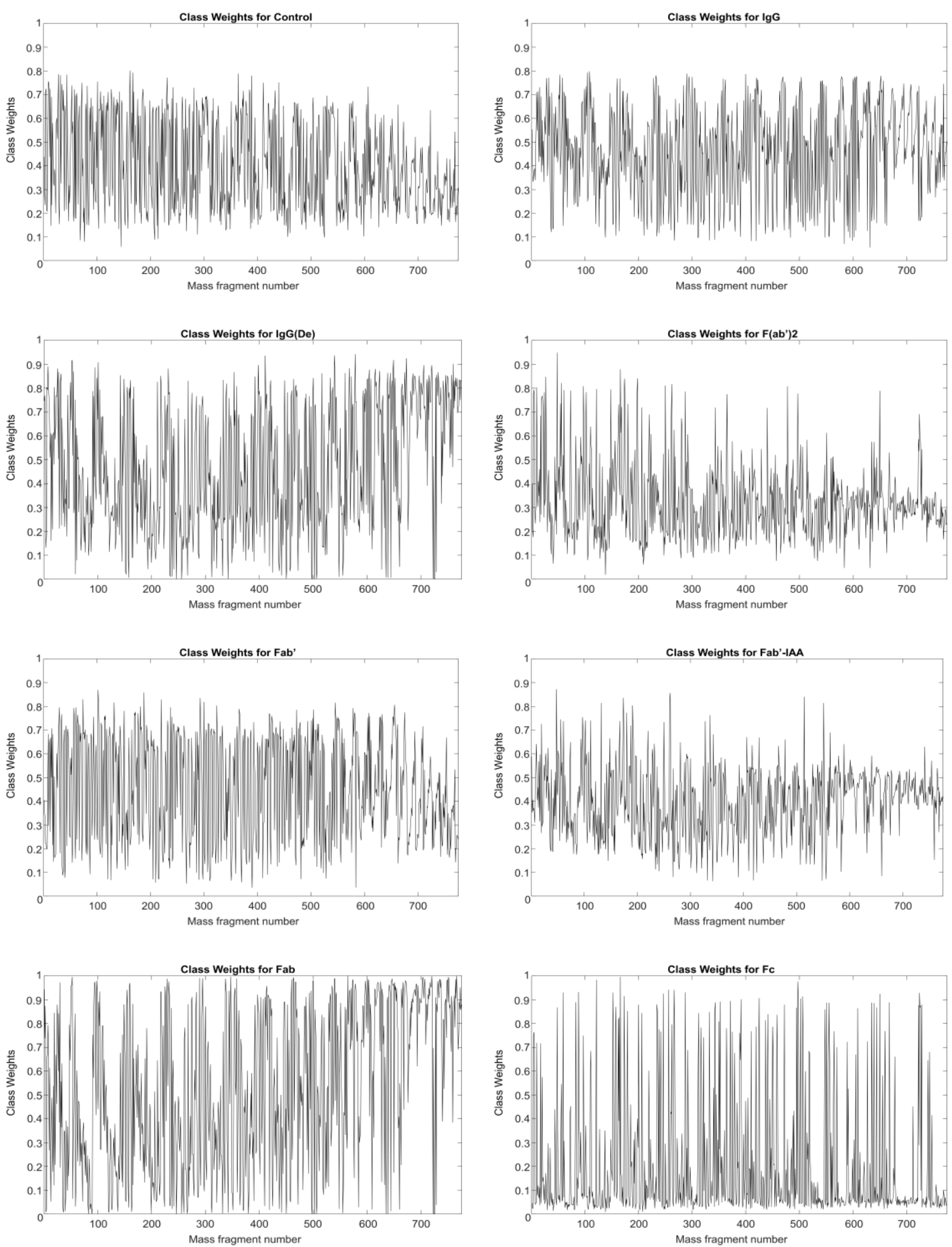

Figure S7. Class weights for 6x6 CP-ANN trained for 100000 epochs with 2 of the $3 \mathrm{Bi}_{3}{ }^{+}$replicate samples with class denoted as fragment type. ToF-SIMS peak list of 775 mass fragments was utilized for the mass range $1-300 \mathrm{~m} / \mathrm{z}$.

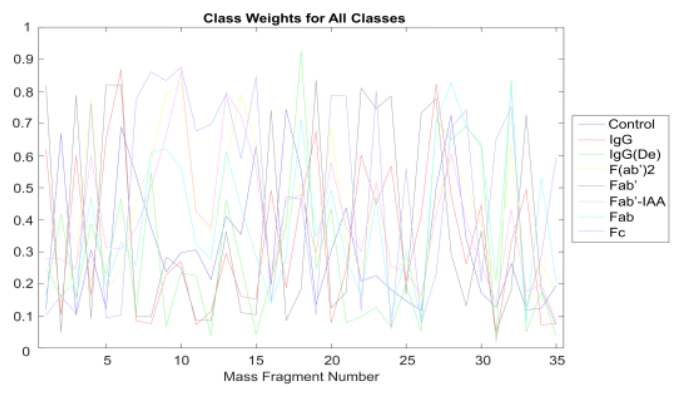



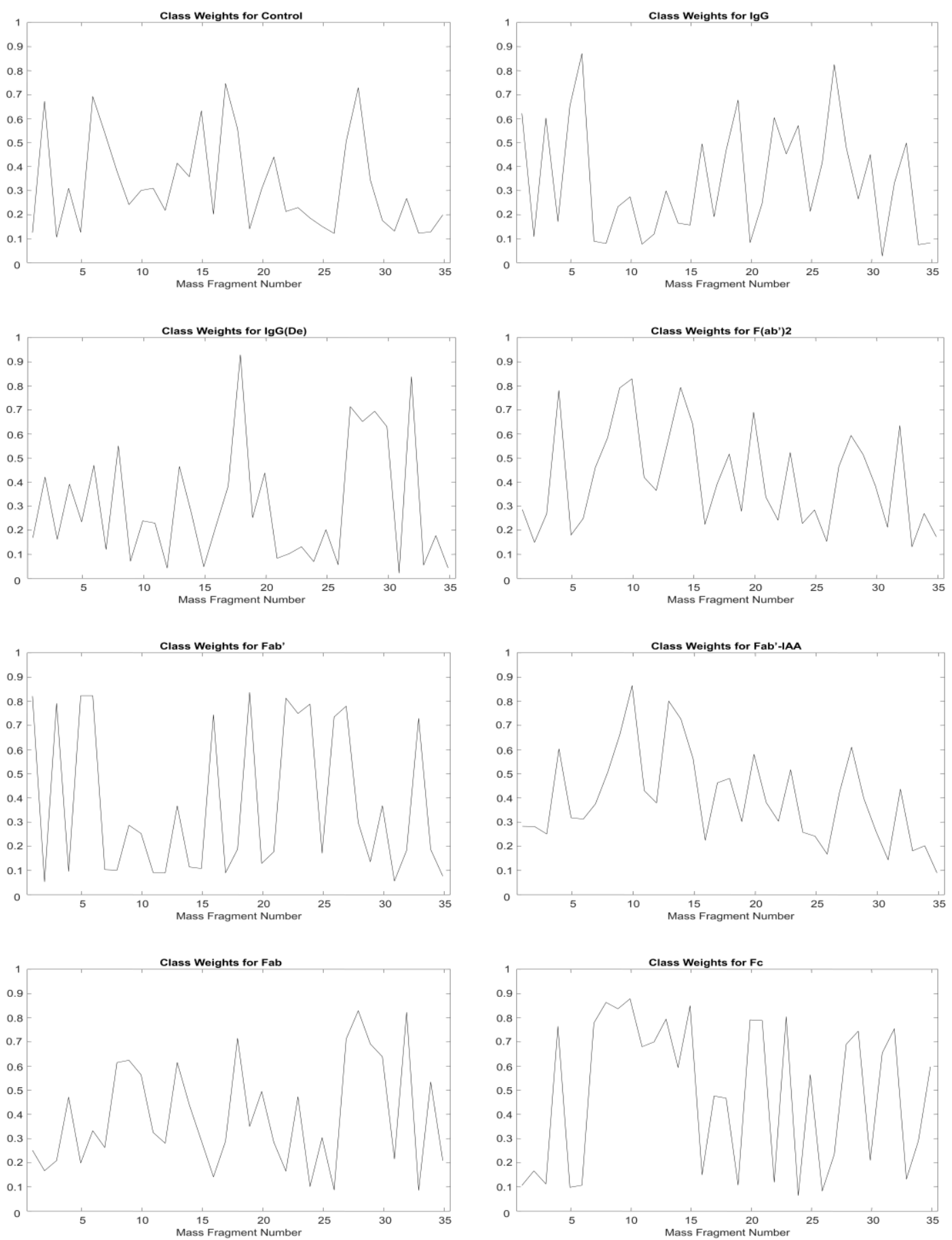

Figure S8. Class weights for $6 \times 6$ CP-ANN trained for 100000 epochs with 2 of the three $\mathrm{Bi}_{3}{ }^{+}$replicate samples with class denoted as fragment type. ToF-SIMS peak list of 35 amino acid mass fragments was utilized. Mass fragment numbers correspond to those in Table S1. 